\section{SARSTEDT}

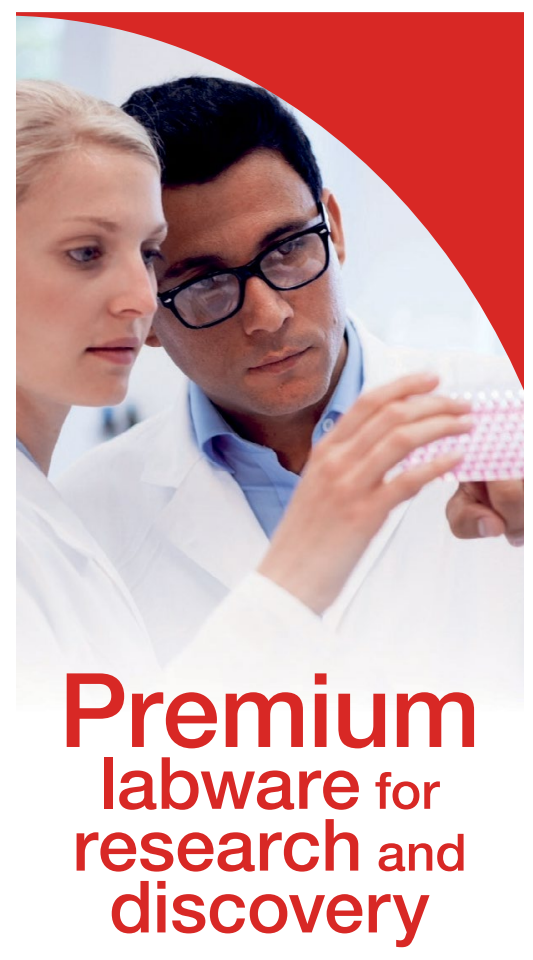

Cell and Tissue Culture

- Three different color coded growth surfaces

- Optimized, user-friendly geometries

- Labeling of all products with LOT number and expiration date

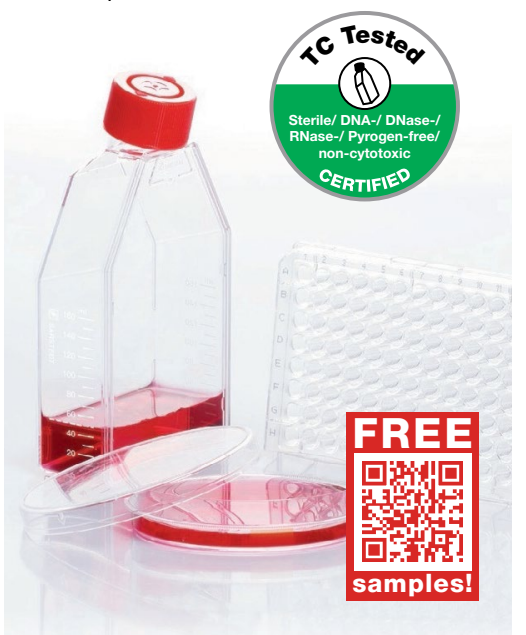

www.sarstedt.com

customerservice@sarstedt.us

STEM CELLS

\title{
A home for brain organoids
}

Inside a mouse brain, human cerebral organoids can show their potential.

A flurry of excitement has surrounded organoids as models of human disease and development. The stemcell-derived three-dimensional structures reconstitute many of the properties found in native tissue. But investigators are also acutely aware of their limitations. Rusty Gage at the Salk Institute and his group have found that a location in the mouse brain supports human organoid development and can overcome some constraints imposed by cell culture.

Organoids tend to mimic young, developing tissues. Both their size and the extent of their development are limited by the absence of blood vessels, which leads to cell death during prolonged culture. The Gage team, led by postdoc Abed Mansour, asked whether organoids would be healthier in the context of a living animal.

Long ago, Gage showed that it was possible to transplant human tissue to the superior colliculus of the rat brain. "I remembered this methodology that I developed and worked on in Sweden 35 years ago," he says, and this prompted him to try organoid tissue. Where other efforts at organoid transplantation failed, Gage's team found that 50-day-old cerebral organoids generated from GFP-expressing human embryonic stem cells could thrive in this location.

The superior colliculus is a special environment that sits atop a rich bed of blood vessels. It takes a steady hand, but anyone can be trained to do the surgery. The key, says Gage, is to "really clean it up so there's no bleeding at all and you haven't nicked the surfaces of the colliculus."

Transplanted organoids were healthy; they showed progressive differentiation of neural and glial cell types, were devoid of cell death, accepted blood vessels and sent axonal projections into host tissue. Organoids imaged through cranial windows showed regular blood flow and calcium spiking. Electrophysiology suggested the maturation of neural networks, and inroducing an optogenetic construct enabled the stimulation of electrical activity in grafted neurons by laser light, with effects in host tissue.

Organoids remained healthy for up to 283 days after transplantation, and were in

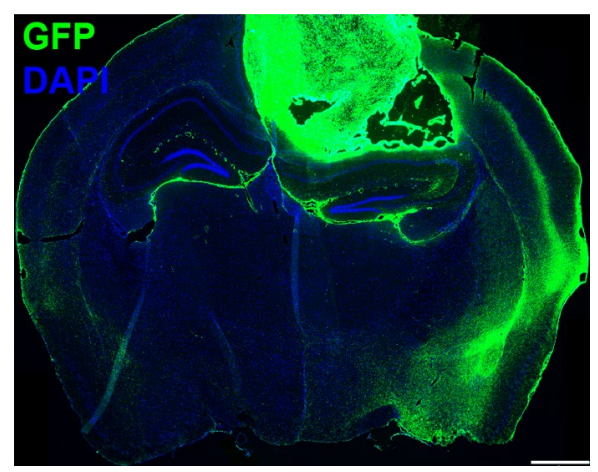

A mouse brain section with GFP-expressing organoid cells 90 days after grafting (nuclei are DAPI-stained). Reproduced with permission from Mansour et al. (2018), Springer Nature.

fact limited only by the short life span of the NOD SCID mouse hosts. Gage is looking to push the current limits of cell maturation by exploring the use of longer-lived strains. The system can also be used to study immune activity, a component that is missing in brain and other organoid studies. The researchers detected scavenging cells called microglia in the graft, though Gage cautions that their origins have not been confirmed.

Transplanted organoids have the potential to provide better disease modeling, but the approach is limited by the need for surgery and organoids of high quality, and by the fact that each animal accommodates only a single organoid. The researchers are now examining the analogous cavity in rat brains, which can accept four or five organoids and thus potentially allow the study of cell interactions across organoids representing different brain regions. They have yet to find any evidence of modulated mouse behavior but are proceeding with caution, given the high degree of functional integration.

\section{Tal Nawy}

Published online: 31 May 2018 https://doi.org/10.1038/s41592-018-0029-8

\section{Research papers}

Mansour, A. A. et al. An in vivo model of functional and vascularized human brain organoids. Nat. Biotechnol. 36, 432-441 (2018). 